\title{
Solvability of the analogs of the problem Tricomi for the mixed type loaded equations with parabolic-hyperbolic operators
}

\section{Umida Baltaeva*}

\section{"Correspondence:}

umida_baltayeva@mail.ru Department of Applied

Mathematics and Mathematics Physics, Urgench State University, Khamid Alimdjan, 14, Urgench, Uzbekistan

\section{Springer}

\begin{abstract}
In this paper the unique solvability of the analog of the Tricomi problem for the third order loaded differential and integro-differential equations with parabolic-hyperbolic operators is proved. The existence of a solution is proved by the reduction to the integral equations with a shift of the second kind. We obtain necessary and sufficient conditions for the existence of a unique solution.
\end{abstract}

Keywords: loaded equation; equations of mixed type; Tricomi problem; Bessel's functions

\section{Introduction}

The first fundamental research on the theory of mixed type equations are the works of F Tricomi, S Gellerstedt, which were published in the 1920s. Due to the research of FI Frankl, IN Vekua, MA Lavrent'ev, and AN Bitsadze, P Germain, R Bader, M Protter, K Morawets, MS Salakhidinov, TD Djuraev, AM Nakhushev, and many other authors, this theory became one of the main directions of the modern theory of partial differential equations.

The necessity of the consideration of the parabolic-hyperbolic type equation was specified in 1956 by Gelffand [1]. He gave an example connected to the movement of the gas in a channel surrounded by a porous environment. Inside the channel the movement of the gas was described by the equation; outside by the diffusion equation.

Recently, loaded equations have been of great interest owing to intensive studying the problems of optimal control of the agro-economical system, of long-term forecasting, and regulating the subsoil waters layer and soil moisture. First, the most general definition of the loaded equation was given by Nakhushev in 1978 [2]. He also gave notions and a detailed classification for different loaded differential, loaded integral, loaded functional equations and considered their numerical applications.

The following equation arises in problems of some mathematical models in problems of particle transfer in the plane-parallel geometry:

$$
\frac{1}{c} \frac{\partial \omega(z)}{\partial z_{3}}+z_{2} \frac{\partial \omega(z)}{\partial z_{1}}+\sigma\left(z_{1}\right) \omega(z)=\frac{\sigma_{3}\left(z_{1}\right)}{2} \int_{-1}^{1} \omega(z) d z_{2}+f(z),
$$

o 2014 Baltaeva; licensee Springer. This is an Open Access article distributed under the terms of the Creative Commons Attribution License (http://creativecommons.org/licenses/by/2.0), which permits unrestricted use, distribution, and reproduction in any medium, provided the original work is properly credited. 
where $\omega(z)=\omega\left(z_{1}, z_{2}, z_{3}\right)$ is the density grain in the point $z_{1}$ at time $z_{3} \geq 0$ flying with speed $c$ under an angle $\theta, \cos \theta=z_{2}$, to the straight line $z_{3}, z_{2}=0$. Moreover, the functions $\sigma\left(z_{1}\right)$, $\sigma_{8}\left(z_{1}\right)$, and $f(z)$ are given.

Also the loaded equation will be the stationary unispeed equation of transport (see [3]),

$$
\frac{1}{\alpha(z)} \frac{\partial \varphi(y, z)}{\partial z_{j}}+\varphi(y, z)=\frac{\lambda}{4 \pi} \int_{|\xi|=1} \theta(z, y, \xi) \varphi(\xi, z) d \xi+F(y, z)
$$

in the phase domain $\left\{(y, z):|y|=\sqrt{y_{1}^{2}+y_{2}^{2}+y_{3}^{2}}=1, z \in \Omega\right\}$. Here $\varphi(y, z)$ is an unknown function, which defines the density of particles flying into the direction of $y,|y|=1$, from the point $z=\left(z_{1}, z_{2}, z_{3}\right)$. The function $\alpha(z)$ is positive and bounded, which characterizes the absorption of the medium; $\lambda$ is the spectral parameter; $\theta(z, y, \xi)$ and $F(y, z)$ are given functions.

Basic questions of the theory of boundary value problems for partial equations are the same for the boundary value problems for the loaded equations. However, the existence of the loaded operator does not always make it possible to apply directly the known theory of boundary value problems.

What puts the considered problems in a class by itself is that the equation of the third order with the most generalized parabolic-hyperbolic and loaded operator is studied. An investigation is in order of the existence and uniqueness of the solution of the stayed boundary value problems for the loaded third order equations mixed type. One investigates the proof of the existence and uniqueness of the solution of the boundary value problem for the loaded differential and integro-differential equations of the mixed type for the analog problems of Tricomi.

\section{Preliminaries}

In this section we give some formulas which will be used in what follows. We note that the regular solutions of some hyperbolic equations are directly connected with RiemannHadamard functions. Therefore, the basic properties such as the decomposition formulas, formulas of the analytical continuation, the formula of the differentiation for the RiemannHadamard function are necessary for studying of the regular solutions [4].

The following function is called the Riemann-Hadamard function [5]:

$$
\begin{aligned}
B(\xi, \eta ; x+y, x-y) & \\
& = \begin{cases}J_{0}\left[\sqrt{\lambda_{2}(\xi-x-y)(\eta-x+y)}\right], & \eta>x+y, \\
J_{0}\left[\sqrt{\lambda_{2}(\xi-x-y)(\eta-x+y)}\right]+J_{0}\left[\sqrt{\lambda_{2}(\eta-x-y)(\xi-x+y)}\right], & \eta<x+y,\end{cases}
\end{aligned}
$$

where $J_{0}[z]$ is the Bessel function [6].

The Riemann-Hadamard function has the following properties [5]:

1. $B\left(\xi, \eta ; \xi_{0}, \eta_{0}\right)$ as function $(\xi, \eta)$ is a solution equation to the conjugate equation $L_{0}^{*}(z)=z_{\xi \eta}+\frac{\lambda}{4} z=0$ and as a function $\left(\xi_{0}, \eta_{0}\right)$ is a solution's characteristic equation $L_{0}(z)=z_{\xi \eta}+\frac{\lambda}{4} z=0$

2. (a) $B_{1 \xi}\left(\xi, \eta ; \xi_{0}, \eta_{0}\right)=0$ for $\eta=\eta_{0}$;

(b) $B_{1 \eta}\left(\xi, \eta ; \xi_{0}, \eta_{0}\right)=0$ for $\xi=\xi_{0}$;

(c) $B_{1}\left(\xi, \eta ; \xi_{0}, \eta_{0}\right)=1$ at $\xi=\xi_{0}$ and $\eta=\eta_{0}$;

3. $\lim _{\varepsilon \rightarrow 0+0}\left[B_{1 \xi}\left(\xi, \xi_{0}+\varepsilon ; \xi_{0}, \eta_{0}\right)-B_{2 \xi}\left(\xi, \xi_{0}-\varepsilon ; \xi_{0}, \eta_{0}\right)\right]=0, \xi \in\left[0, \xi_{0}\right]$ 
4. $B_{2 \eta}\left(\xi, \eta ; \xi_{0}, \eta_{0}\right)-B_{2 \xi}\left(\xi, \eta ; \xi_{0}, \eta_{0}\right)=0$ at $\eta=\xi$, where $B_{1}$ and $B_{2}$ denote the Riemann-Hadamard functions for $\eta>\xi_{0}$ and $\eta<\xi_{0}$.

\section{Formulating the problem and main functional relations}

Consider the equation

$$
0=\frac{\partial}{\partial x} \begin{cases}u_{x x}-u_{y}-\lambda_{1} u-\mu_{1} u(x, 0), & y>0, \\ u_{x x}-u_{y y}+\lambda_{2} u-\mu_{2} u(x, 0), & y<0,\end{cases}
$$

in the domain $\Omega$.

$\Omega$ is a simple connected domain located in the plane of independent variables $x$ and $y$, bounded by $y>0$ with segments $A A_{0}, B B_{0}, A_{0} B_{0}\left(A(0,0), B(1,0), A_{0}(0,1), B_{0}(1,1)\right)$ and by $y<0$ with characteristics $A C: x+y=0, B C: x-y=1$ of (1).

We use the following:

$$
\Omega_{1}=\Omega \cap\{y>0\}, \quad \Omega_{2}=\Omega \cap\{y<0\}, \quad I=\{(x, y): 0<x<1, y=0\} .
$$

In (1) $\lambda_{k}, \mu_{k}(k=1,2)$ are given real parameters.

We investigate the following problem.

Problem $\mathbf{T}_{0}$ Find a function $u(x, y)$ satisfying the conditions:

(1) $u(x, y) \in C(\bar{\Omega}) \cap C^{1}\left(\Omega \cup A C \cup A A_{0}\right) \cap C_{x, y}^{3,1}\left(\Omega_{1}\right) \cap C_{x, y}^{3,2}\left(\Omega_{2}\right)$;

(2) $u(x, y)$ satisfies equation (1) in $\Omega_{1}$ and $\Omega_{2}$;

(3) $u(x, y)$ satisfies the boundary conditions:

$$
\begin{aligned}
& \left.u(x, y)\right|_{A A_{0}}=\varphi_{1}(y),\left.\quad u_{x}(x, y)\right|_{A A_{0}}=\varphi_{2}(y), \\
& \left.u(x, y)\right|_{B B_{0}}=\varphi_{3}(y), \quad 0 \leq y \leq 1, \\
& \left.u(x, y)\right|_{A C}=\psi_{1}(x),\left.\quad \frac{\partial u(x, y)}{\partial n}\right|_{A C}=\psi_{2}(x), \quad 0 \leq x \leq \frac{1}{2},
\end{aligned}
$$

where $n$ is the interior normal, $\varphi_{1}(y), \varphi_{2}(y), \varphi_{3}(y), \psi_{1}(x)$, and $\psi_{2}(x)$ are given real-valued functions, such that $\varphi_{1}(0)=\psi_{1}(0)$.

Let $\lambda_{1}>0, \lambda_{2}>0, \mu_{k} \neq 0, k=1,2$.

Equation (1) can be written as [7]

$$
\begin{array}{ll}
u_{x x}-u_{y}-\lambda_{1} u-\mu_{1} u(x, 0)=w_{1}(y), & y>0, \\
u_{x x}-u_{y y}+\lambda_{2} u-\mu_{2} u(x, 0)=w_{2}(y), & y<0,
\end{array}
$$

here $w_{1}(y), w_{2}(y)$ are arbitrary continuous functions.

We use the following:

$$
\begin{aligned}
& u(x, \pm 0)=\tau(x), \quad(x, 0) \in \bar{I}, \\
& u_{y}(x, \pm 0)=v(x), \quad(x, 0) \in I .
\end{aligned}
$$


It is known that every regular solution of (5) in $\Omega_{2}$, with the first boundary condition (3) and (7) is represented as [5]

$$
\begin{aligned}
u(x, y)= & \int_{0}^{x+y} v(\xi) J_{0}\left[\sqrt{\lambda_{2}(\xi-x-y)(\xi-x+y)}\right] d \xi \\
& +\psi_{1}(0) J_{0}\left[\sqrt{\lambda_{2}\left(x^{2}-y^{2}\right)}\right]+\frac{1}{2} \int_{0}^{x-y} \psi_{1}^{\prime}\left(\frac{\eta}{2}\right) B(0, \eta ; x+y, x-y) d \eta \\
& +\frac{1}{4} \int_{0}^{x+y} d \xi \int_{\xi}^{x-y} B(\xi, \eta ; x+y, x-y)\left(w_{2}\left(\frac{\xi-\eta}{2}\right)+\mu_{2} \tau\left(\frac{\xi+\eta}{2}\right)\right) d \eta,
\end{aligned}
$$

where $B(\xi, \eta ; x+y, x-y)$ is the Riemann-Hadamard function [5], $J_{0}[z]$ is the Bessel function [6].

Using the second condition of (3), considering property 2,3 of $B\left(\xi, \eta ; \xi_{1}, \eta_{1}\right)$ we get

$$
\begin{aligned}
\int_{0}^{x}\left(w_{2}(-t)+\mu_{2} \tau(t)\right) d t= & \sqrt{2} \psi_{2}(x)+\frac{\lambda_{2}}{4} \int_{0}^{2 x}(2 x-\eta) \psi_{1}^{\prime}\left(\frac{\eta}{2}\right) d \eta \\
& +\lambda_{2} x \psi_{1}(0)-2 v(0) .
\end{aligned}
$$

Differentiating (9) with respect to $x$ and replace variables with $y\left(-\frac{1}{2} \leq y \leq 0\right)$, bearing in mind $v(0)=\frac{1}{\sqrt{2}} \psi_{2}(0)$, we find the function $w_{2}(y)$ :

$$
w_{2}(y)=-\mu_{2} \tau(-y)-\sqrt{2} \psi_{2}^{\prime}(-y)+\lambda_{2} \psi_{1}(-y), \quad-\frac{1}{2} \leq y \leq 0 .
$$

Substituting (8) into (6) we have

$$
\begin{aligned}
\tau(x)= & \int_{0}^{x} v(\xi) J_{0}\left[\sqrt{\lambda_{2}}(x-\xi)\right] d \xi+\int_{0}^{x} \psi_{1}^{\prime}\left(\frac{\eta}{2}\right) J_{0}\left[\sqrt{\lambda_{2} x(x-\eta)}\right] d \eta \\
& +\psi_{1}(0) J_{0}\left[\sqrt{\lambda_{2}} x\right]+\frac{1}{2} \int_{0}^{x} d \xi \int_{\xi}^{x} J_{0}\left[\sqrt{\lambda_{2}(\xi-x)(\eta-x)}\right]\left(\mu_{2} \tau\left(\frac{\xi+\eta}{2}\right)\right. \\
& \left.+w_{2}\left(\frac{\xi-\eta}{2}\right)\right) d \eta .
\end{aligned}
$$

Substituting into (11) the expression of $w_{2}(y)$ by (10) and after some transformation we find the main functional relation between the function $\tau(x)$ and $v(x)$ on $A B$ in the domain $\Omega_{2}$ :

$$
\begin{aligned}
\tau(x)= & \int_{0}^{x} v(\xi) J_{0}\left[\sqrt{\lambda_{2}}(x-\xi)\right] d \xi+\mu_{2} \int_{0}^{x} s(x, t) \tau(t) d t \\
& -\mu_{2} \int_{0}^{\frac{x}{2}} \tau(t) \int_{0}^{x-2 t} J_{0}\left[\sqrt{\lambda_{2}(\xi-x)(\xi+2 t-x)}\right] d \xi d t \\
& +\int_{0}^{\frac{x}{2}}\left(\lambda_{2} \psi_{1}(t)-\sqrt{2} \psi_{2}^{\prime}(t)\right) \int_{0}^{x-2 t} J_{0}\left[\sqrt{\lambda_{2}(\xi-x)(\xi-x+2 t)}\right] d \xi d t \\
& +\int_{0}^{x} \psi_{1}^{\prime}\left(\frac{t}{2}\right) J_{0}\left[\sqrt{\lambda_{2} x(x-t)}\right] d t+\psi_{1}(0) J_{0}\left[\sqrt{\lambda_{2}} x\right]
\end{aligned}
$$


here

$$
s(x, t)= \begin{cases}\int_{0}^{t} J_{0}\left[\sqrt{\lambda_{2}(\xi-x)(2 t-\xi-x)}\right] d \xi, & 0<t \leq \frac{x}{2}, \\ \int_{2 t-x}^{t} J_{0}\left[\sqrt{\lambda_{2}(\xi-x)(2 t-\xi-x)}\right] d \xi, & \frac{x}{2} \leq t<x .\end{cases}
$$

Passing to the limit in (4) at $y \rightarrow+0$ considering $u(x, y) \in C^{1}(\Omega)$, (6), (7) we have [7]

$$
\tau^{\prime \prime}(x)-v(x)-\left(\lambda_{1}+\mu_{1}\right) \tau(x)=w_{1}(0)
$$

where $w_{1}(0)$ is an unknown constant, which will be found later on.

\section{Main results}

Theorem 1 If

$$
\begin{aligned}
& \varphi_{1}(y) \in C^{1}[0,1], \quad \varphi_{2}(y) \in C[0,1] \cap C^{1}(0,1), \quad \varphi_{3}(y) \in C^{1}[0,1], \\
& \psi_{1}(x) \in C^{1}\left[0, \frac{1}{2}\right] \cap C^{3}\left(0, \frac{1}{2}\right), \quad \psi_{2}(x) \in C^{1}\left[0, \frac{1}{2}\right] \cap C^{3}\left(0, \frac{1}{2}\right),
\end{aligned}
$$

then there exists a unique solution to the problem $\mathrm{T}_{0}$.

Proof From (14) and (12), bearing in mind $u(x, y) \in C^{1}(\Omega)$,

$$
\tau(0)=\varphi_{1}(0), \quad \tau^{\prime}(0)=\varphi_{2}(0), \quad \tau(\eta)=\varphi_{1}(0)+\int_{0}^{\eta} \tau^{\prime}(t) d t,
$$

using integration by parts, after some transformations we have

$$
\tau^{\prime}(x)-\int_{0}^{x} K_{1}(x, t) \tau^{\prime}(t) d t-\mu_{2} \int_{0}^{\frac{x}{2}} K_{2}(x, t) \tau^{\prime}(t) d t=F_{1}(x)+w_{1}(0) F_{2}(x),
$$

here

$$
\begin{aligned}
K_{1}(x, t)= & 1+\sqrt{\lambda_{2}} \bar{J}_{1}\left[\sqrt{\lambda_{2}}(x-t)\right]+\int_{t}^{x}\left(\left(\lambda_{1}+\mu_{1}\right) J_{0}\left[\sqrt{\lambda_{2}}(x-\xi)\right]-\mu_{2} s(x, \xi)\right) d \xi, \\
K_{2}(x, t)= & \int_{t}^{\frac{x}{2}} d s \int_{0}^{x-2 s} J_{0}\left[\sqrt{\lambda_{2}(\xi-x)(\xi-x+2 s)}\right] d \xi \\
F_{1}(x)= & -\int_{0}^{x} \psi_{1}^{\prime}\left(\frac{t}{2}\right) J_{0}\left[\sqrt{\lambda_{2} x(x-t)}\right] d t-\int_{0}^{\frac{x}{2}}\left(\lambda_{2} \psi_{1}(t)+\sqrt{2} \psi_{2}^{\prime}(t)-\varphi_{1}(0)\right) d t \\
& \times \int_{0}^{x-2 t} J_{0}\left[\sqrt{\lambda_{2}(\xi-x)(\xi-x+2 t)}\right] d \xi+\varphi_{2}(0) J_{0}\left[\sqrt{\lambda_{2}} x\right]+\varphi_{1}(0) \\
& \times\left\{1-J_{0}\left[\sqrt{\lambda_{2} x}\right]+\int_{0}^{x}\left[\left(\lambda_{1}+\mu_{1}\right) J_{0}\left[\sqrt{\lambda_{2}}(x-t)\right]-\mu_{2} s(x, t)\right] d t\right\} \\
F_{2}(x)= & \int_{0}^{x} J_{0}\left[\sqrt{\lambda_{2}}(x-t)\right] d t .
\end{aligned}
$$

Equation (18) is the Volterra type integral equation of the second kind with shift [8], $s(x, \xi)$ is defined by (13). 
Setting

$$
\Phi_{1}(x)=F_{1}(x)+\mu_{2} \int_{0}^{\frac{x}{2}} K_{2}(x, t) \tau^{\prime}(t) d t+w_{1}(0) F_{2}(x),
$$

equation (18) can be written as [9]

$$
\tau^{\prime}(x)-\int_{0}^{x} K_{1}(x, t) \tau^{\prime}(t) d t=\Phi_{1}(x)
$$

From the representations of the functions $K_{1}(x, t)$ and $\Phi_{1}(x)$, using some properties of the Bessel function [6] based on the general theory of integral equation [7], one can easily ensure that (24) has a unique solution, which is represented as

$$
\tau^{\prime}(x)=\Phi_{1}(x)+\int_{0}^{x} R_{1}(x, t) \Phi_{1}(t) d t
$$

where $R_{1}(x, t)$ is the resolvent kernel of $K_{1}(x, t)$.

Substituting (23) into (25), after some transformations we obtain a Volterra type integral equation of the second kind with shift,

$$
\begin{aligned}
& \tau^{\prime}(x)-\mu_{2} \int_{0}^{\frac{x}{2}} \bar{K}_{2}(x, t) \tau^{\prime}(t) d t=\Phi_{2}(x) \\
& \bar{K}_{2}(x, t)=K_{2}(x, t)+\int_{2 t}^{x} R_{1}(x, t) K_{2}(s, t) d s \\
& \Phi_{2}(x)=F_{1}(x)+\int_{0}^{x} R_{1}(x, t) F_{1}(t) d t+w_{1}(0)\left(F_{2}(x)+\int_{0}^{x} R_{1}(x, t) F_{2}(t) d t\right) .
\end{aligned}
$$

Taking into consideration (25), (28), (29) enclose

$$
\left|\bar{K}_{2}(x, t)\right| \leq \text { const }, \quad\left|\Phi_{2}(x)\right| \leq \text { const. }
$$

Using the method of successive approximations in (26), we examine the following sequence:

$$
\begin{aligned}
& \tau_{0}^{\prime}(x)=\Phi_{2}(x) \\
& \tau_{1}^{\prime}(x)=\Phi_{2}(x)+\mu_{2} \int_{0}^{\frac{x}{2}} \bar{K}_{2}(x, t) \Phi_{2}(t) d t, \\
& \tau_{2}^{\prime}(x)=\Phi_{2}(x)+\mu_{2} \int_{0}^{\frac{x}{2}} \bar{K}_{2}(x, t) \tau_{1}^{\prime}(t) d t, \\
& \ldots \\
& \tau_{n}^{\prime}(x)=\Phi_{2}(x)+\mu_{2} \int_{0}^{\frac{x}{2}} \bar{K}_{2}(x, t) \tau_{n-1}^{\prime}(t) d t .
\end{aligned}
$$


For proving the existence of the solution (26) it is enough to prove the uniform convergence $\left\{\tau_{n}^{\prime}(x)\right\}_{n=1}^{\infty}$ which is equivalent to convergence as regards the following series [10]:

$$
\tau_{0}^{\prime}(x)+\sum_{n=1}^{\infty}\left[\tau_{n}^{\prime}(x)-\tau_{n-1}^{\prime}(x)\right]
$$

Regarding $\max \left|\bar{K}_{2}(x, t)\right|=M, \max _{x \in[0,1]}|f(x)|=m$, estimate

$$
\begin{aligned}
& \left|\tau_{0}^{\prime}(x)\right| \leq m, \quad\left|\tau_{1}^{\prime}(x)-\tau_{0}^{\prime}(x)\right| \leq\left|\mu_{2} \int_{0}^{\frac{x}{2}} \bar{K}_{2}(x, t) \tau_{0}^{\prime}(t) d t\right| \leq\left|\mu_{2}\right| m M \frac{x}{2}, \\
& \left|\tau_{2}^{\prime}(x)-\tau_{1}^{\prime}(x)\right| \leq\left|\mu_{2} \int_{0}^{\frac{x}{2}} \bar{K}_{2}(x, t)\left[\tau_{1}^{\prime}(t)-\tau_{0}^{\prime}(t)\right] d t\right| \leq\left|\mu_{2}\right|^{2} m M^{2} \frac{x^{2}}{2 ! 2^{3}}, \\
& \left|\tau_{n}^{\prime}(x)-\tau_{n-1}^{\prime}(x)\right| \leq\left|\mu_{2} \int_{0}^{\frac{x}{2}} \bar{K}_{2}(x, t)\left[\tau_{n-1}^{\prime}(t)-\tau_{n-2}^{\prime}(t)\right] d t\right| \leq\left|\mu_{2}\right|^{n} m M^{n} \cdot \frac{x^{n}}{n ! 2^{\frac{n(n+1)}{2}}} .
\end{aligned}
$$

Consequently it is a sequential estimation of the series of (30) for each value $\mu_{2}$ with absolute and uniform convergence and the function $\tau^{\prime}(x)=\lim _{n \rightarrow \infty} \tau_{n}^{\prime}(x)$ is a solution of $(26)$ in $[0,1]$.

For proving the unique solution equation $(26)$ in class $C[0,1]$ it is enough to prove that the corresponding homogeneous equation has only a trivial solution in this class.

Let us consider the corresponding homogeneous equation,

$$
\chi_{1}^{\prime}(x)-\mu_{2} \int_{0}^{x / 2} \bar{K}_{2}(x, t) \chi_{1}^{\prime}(t) d t=0
$$

and denote $\max _{x \in[0,1]} \chi_{1}^{\prime}(x)=m^{*}$. Consider $\chi_{1}^{\prime}(x)$, and we get the following inequality:

$$
\begin{aligned}
& \left|\chi_{1}^{\prime}(x)\right| \leq\left|\mu_{2}\right| \int_{0}^{x / 2}\left|\bar{K}_{2}(x, t)\right|\left|\chi_{1}^{\prime}(t)\right| d t \leq\left|\mu_{2}\right| M m^{*} x, \\
& \left|\chi_{1}^{\prime}(x)\right| \leq\left|\mu_{2}\right| \int_{0}^{x / 2}\left|\bar{K}_{2}(x, t)\right|\left|\chi_{1}^{\prime}(t)\right| d t \leq\left|\mu_{2}\right|^{2} M^{2} m^{*} \frac{x^{2}}{2 ! 2^{3}}, \\
& \ldots \\
& \left|\chi_{1}^{\prime}(x)\right| \leq\left|\mu_{2}\right| \int_{0}^{x / 2}\left|\bar{K}_{2}(x, t)\right|\left|\chi_{1}^{\prime}(t)\right| d t \leq\left|\mu_{2}\right|^{n} M^{n} m^{*} \frac{x^{n}}{n ! 2^{\frac{n(n+1)}{2}}} .
\end{aligned}
$$

Reducing the foregoing estimate for $n \rightarrow \infty$ it follows that $\chi_{1}^{\prime}(x)=0$.

Thus the proof of (26) exists as a unique solution which is equivalent with problem $\mathrm{T}_{0}$. Taking account of (27), (28), (29) we get the solution equation (26) as

$$
\begin{aligned}
\tau(x)= & \varphi_{1}(0)+\int_{0}^{x}\left[\bar{F}_{1}(t)+\mu_{2} \int_{0}^{\frac{t}{2}} R_{2}\left(t, s, \mu_{2}\right) \bar{F}_{1}(s) d s\right] d t \\
& +w_{1}(0) \int_{0}^{x}\left[\bar{F}_{2}(t)+\mu_{2} \int_{0}^{\frac{t}{2}} R_{2}\left(t, s, \mu_{2}\right) \bar{F}_{2}(s) d s\right] d t,
\end{aligned}
$$

where

$$
\bar{F}_{1}(x)=F_{1}(x)+\int_{0}^{x} R_{1}(x, t) F_{1}(t) d t, \quad \bar{F}_{2}(x)=F_{2}(x)+\int_{0}^{x} R_{1}(x, t) F_{2}(t) d t,
$$


$R_{2}\left(x, t, \mu_{2}\right)$ is the resolvent kernel of $\bar{K}_{2}(x, t)$. Here, by virtue of (15), (16), with regard to (29) we enclose the function $\tau(x)$ in the class $C^{1}[0,1] \cap C^{3}(0,1)$.

After finding $\tau(x)$ according to the condition $\tau(1)=\varphi_{3}(0)$, we can find the value of $w_{1}(0)$. Using relation (11), (14) and taking into account (31) we uniquely define the functions $w_{2}(y)$ and $v(x)$.

After the determination of $v(x)$ and $w_{2}(y)$ the solution of problem $\mathrm{T}_{0}$ in the domain $\Omega_{2}$ would be reconstructed (reconstruction) just as the solution of the Cauchy [4] problem or Darboux in (8) for (5).

It is obvious that the problem for (1) in $\Omega_{1}$ is an undetermined real function which is equivalent to the following problem $T^{*}$ for (4) with the boundary conditions (2) and $u(x,+0)=\tau(x)$.

Introduce the new unknown function $z(x, y)$,

$$
u(x, y)=z(x, y) \cdot e^{-\lambda_{1} y} .
$$

Therefore, the boundary value problem in $\Omega_{1}$ is reduced to the following boundary value problem, which is called problem $T^{*}$ in $\Omega_{1}$ :

$$
\begin{aligned}
& \frac{\partial}{\partial x}\left(z_{x x}-z_{y}\right)=F(x, y), \\
& \left.z(x, y)\right|_{A A_{0}}=e^{\lambda_{1} y} \varphi_{1}(y),\left.\quad z_{x}(x, y)\right|_{A A_{0}}=e^{\lambda_{1} y} \varphi_{2}(y),\left.\quad z(x, y)\right|_{B B_{0}}=e^{\lambda_{1} y} \varphi_{3}(y), \\
& z(x, 0)=\tau(x),
\end{aligned}
$$

here $F(x, y)=\mu_{1} e^{\lambda_{1} y} \tau^{\prime}(x), \tau^{\prime}(x)$ is defined by $(31)$.

The proof of the unique solvability of problem $T^{*}$ can be found in [7]. Hence, problem $\mathrm{T}_{0}$ has also unique solvability. Theorem 1 is proved.

Remark Analogously we can prove unique solvability for problem $\mathrm{T}_{0}$ where $\lambda_{1}>0, \lambda_{2}<0$ and $\mu_{k} \neq 0, k=1,2$.

\section{Problem $\mathrm{T}_{1}$ for integro-differential equation}

Let $\Omega$ be a simple connected domain located in the plane of independent variables $x$ and $y$, bounded by $y>0$ with segments $A A_{0}, B B_{0}, A_{0} B_{0}\left(A(0,0), B(1,0), A_{0}(0,1), B_{0}(1,1)\right)$ and by $y<0$ with the characteristics

$$
A C: \xi=x+y=0, \quad B C: \eta=x-y=1,
$$

of the equation

$$
0=\frac{\partial}{\partial x} \begin{cases}u_{x x}-u_{y}-\lambda_{1} u-\sum_{i=1}^{n} a_{i}(x, y) D_{0 x}^{\alpha_{i}} u(x, 0), & y>0, \\ u_{x x}-u_{y y}-\lambda_{2} u-\sum_{i=1}^{n} b_{i}(x, y) D_{0 \xi}^{\beta_{i}} u(\xi, 0), & y<0, \xi=x+y,\end{cases}
$$

here $\lambda_{1}, \lambda_{2}$ are given real parameters, $D_{o x}^{\gamma_{i}}\left(\gamma_{i}=\alpha_{i}, \beta_{i}\right)$ are integro-differential operators [2, $11]$.

We use the following:

$$
\Omega_{1}=\Omega \cap\{y>0\}, \quad \Omega_{2}=\Omega \cap\{y<0\}, \quad I=\{(x, y): 0<x<1, y=0\} .
$$


Problem $\mathbf{T}_{1}$ Find a function $u(x, y)$ satisfying the conditions:

(1) $u(x, y) \in C(\bar{\Omega}) \cap C_{x, y}^{3,1}\left(\Omega_{1}\right) \cap C_{x, y}^{3,2}\left(\Omega_{2}\right)$;

(2) $u_{x}\left(u_{y}\right)$ is continuous up to $A A_{0} \cup A B \cup A C(A B \cup A C)$;

(3) the sewing condition

$$
u_{y}(x,-0)=u_{y}(x,+0), \quad 0<x<1
$$

(4) the boundary conditions

$$
\begin{aligned}
& \left.u(x, y)\right|_{A A_{0}}=\varphi_{1}(y),\left.\quad u_{x}(x, y)\right|_{A A_{0}}=\varphi_{2}(y), \\
& \left.u(x, y)\right|_{B B_{0}}=\varphi_{3}(y), \quad 0 \leq y \leq 1, \\
& \left.u(x, y)\right|_{A C}=\psi_{1}(x),\left.\quad \frac{\partial u(x, y)}{\partial n}\right|_{A C}=\psi_{2}(x), \quad 0 \leq x \leq \frac{1}{2},
\end{aligned}
$$

where $n$ is the interior normal, $\varphi_{1}(y), \varphi_{2}(y), \varphi_{3}(y), \psi_{1}(x)$, and $\psi_{2}(x)$ are given real-valued functions, moreover, $\varphi_{1}(0)=\psi_{1}(0)=0$.

Theorem 2 If $\lambda_{1}>0, \lambda_{2}>0, a_{i}(x, y) \geq 0,(x, y) \in \Omega_{1}, b_{i}(x, y) \geq 0,(x, y) \in \Omega_{2}$, and

$$
\begin{aligned}
& a_{i}(x, y) \in C^{1}\left(\bar{\Omega}_{1}\right) \cap C^{2}\left(\Omega_{1}\right), \quad b_{i}(x, y) \in C^{1}\left(\bar{\Omega}_{2}\right) \cap C^{3}\left(\Omega_{2}\right) \quad(i=\overline{1, n}), \\
& \varphi_{1}(y), \varphi_{3}(y) \in C^{1}[0,1], \quad \varphi_{2}(y) \in C[0,1] \cap C^{1}(0,1) \\
& \psi_{1}(x) \in C^{1}\left[0, \frac{1}{2}\right] \cap C^{3}\left(0, \frac{1}{2}\right), \quad \psi_{2}(x) \in C^{1}\left[0, \frac{1}{2}\right] \cap C^{2}\left(0, \frac{1}{2}\right)
\end{aligned}
$$

then there exists a unique solution to problem $\mathrm{T}_{1}$.

Proof of Theorem 2 Equation (32) can be written as [12]

$$
\begin{aligned}
& u_{x x}-u_{y}-\lambda_{1} u-\sum_{i=1}^{n} a_{i}(x, y) D_{o x}^{\alpha_{i}} u(x, 0)=w_{1}(y), \\
& u_{x x}-u_{y y}-\lambda_{2} u-\sum_{i=1}^{n} b_{i}(x, y) D_{o \xi}^{\beta_{i}} u(\xi, 0)=w_{2}(y),
\end{aligned}
$$

here $w_{1}(y), w_{2}(y)$ are arbitrary continuous functions.

We use the following:

$$
\begin{aligned}
& u(x,-0)=\tau(x), \quad(x, 0) \in \bar{I}, \\
& u_{y}(x,-0)=v(x), \quad(x, 0) \in I .
\end{aligned}
$$

It is well known that every regular solution of (40) in $\Omega_{2}$, with the first boundary condition (35) and (42) is represented as in [4].

The solution of (40) in domain $\Omega_{2}$, with first boundary conditions (35) and (42) exists (condition (36) guarantees the existence of the Riemann-Hadamard functions for the 
equation), a unique solution, representable in the form of

$$
\begin{aligned}
u(x, y)= & \int_{0}^{x+y} v(\xi) J_{0}\left[\sqrt{\lambda_{2}(\xi-x-y)(\xi-x+y)}\right] d \xi \\
& +\psi_{1}(0) J_{0}\left[\sqrt{\lambda_{2}\left(x^{2}-y^{2}\right)}\right]+\frac{1}{2} \int_{0}^{x-y} \psi_{1}^{\prime}\left(\frac{\eta}{2}\right) B(0, \eta ; x+y, x-y) d \eta \\
& +\frac{1}{4} \int_{0}^{x+y} d \xi \int_{\xi}^{x-y} B(\xi, \eta ; x+y, x-y) w_{2}\left(\frac{\xi-\eta}{2}\right) d \eta \\
& +\frac{1}{4} \int_{0}^{x+y} D_{o \xi}^{\beta_{i}} \tau(\xi) d \xi \int_{\xi}^{x-y} B(\xi, \eta ; x+y, x-y) b_{i}\left(\frac{\xi+\eta}{2}, \frac{\eta-\xi}{2}\right) d \eta,
\end{aligned}
$$

where $B(\xi, \eta ; x+y, x-y)$ is a Riemann-Hadamard function [4], $J_{0}[z]$ is a Bessel function [6], and for the index we have the implied summation from 1 to $n$.

Satisfying (43), the second boundary condition of (35), and taking account of

$$
\tau(0)=\psi_{1}(0)=0, \quad v(0)=\frac{1}{\sqrt{2}} \psi_{2}(0)
$$

and property 2, 3, and 4 the Riemann-Hadamard functions, after some transformation we find $w_{2}(y)$ :

$$
w_{2}(y)=-\sqrt{2} \psi_{2}^{\prime}(-y)+\lambda_{2} \psi_{1}(-y)+\lambda_{2} \psi_{1}(0), \quad-\frac{1}{2} \leq y \leq 0 .
$$

By virtue of (41) from (43) we have

$$
\begin{aligned}
\tau(x)= & \int_{0}^{x} v(\xi) J_{0}\left[\sqrt{\lambda_{2}}(x-\xi)\right] d \xi+\int_{0}^{x} \psi_{1}^{\prime}\left(\frac{\eta}{2}\right) J_{0}\left[\sqrt{\lambda_{2} x(x-\eta)}\right] d \eta \\
& +\psi_{1}(0) J_{0}\left[\sqrt{\lambda_{2}} x\right]+\frac{1}{2} \int_{0}^{x} d \xi \int_{\xi}^{x} w_{2}\left(\frac{\xi-\eta}{2}\right) d \eta \\
& +\frac{1}{2} \int_{0}^{x} D_{0 \xi}^{\beta_{i}} \tau(\xi) d \xi \int_{\xi}^{x} J_{0}\left[\sqrt{\lambda_{2}(\xi-x)(\eta-x)}\right] b_{i}\left(\frac{\xi+\eta}{2}, \frac{\eta-\xi}{2}\right) d \eta .
\end{aligned}
$$

Here $w_{2}(y)$ is determined by (45).

Passing to the limit in (39), $y \rightarrow+0$, taking account of (2), (41), and (42), we obtain a second functional relation between the function $\tau(x)$ and $v(x)$, transferred from the $\Omega_{1}$ to $A B$ :

$$
\tau^{\prime \prime}(x)-v(x)-\lambda_{1} \tau(x)-\sum_{i=1}^{n} a_{i}(x, 0) D_{0 x}^{\alpha_{i}} \tau(x)=w_{1}(0)
$$

where $w_{1}(0)$ is an unknown constant to be defined.

Except the function $v(x)$ in (46) and (47), in view of the sewing condition, we obtain an integral equation with a shift [8] with respect to $\tau^{\prime}(x)$ :

$$
\tau^{\prime}(x)-\int_{0}^{x} K_{n}(x, t) \tau^{\prime}(t) d t=\omega_{1}(0) f_{1}(x)+f_{2}(x)
$$


here

$$
\begin{aligned}
K_{1}(x, t)= & +\sqrt{\lambda_{2}} J_{0}\left[\sqrt{\lambda_{2}}(x-t)\right]+\lambda_{1} \int_{t}^{x} J_{0}\left[\sqrt{\lambda_{2}}(x-\xi)\right] d \xi \\
& -\frac{1}{\alpha_{i} \Gamma\left(-\alpha_{i}\right)}(x-t)^{1-\alpha_{i}} \int_{0}^{1} \frac{A_{i} J_{0}\left[\sqrt{\lambda_{2}}(x-t)(1+v)\right]}{v^{\alpha_{i}}} d v \\
& +\frac{1}{\beta_{i} \Gamma\left(-\beta_{i}\right)}(x-t)^{1-\beta_{i}} \int_{0}^{1} \frac{K_{0}(x, t+(x-t) v)}{v^{\beta_{i}}} d v, \quad \alpha_{i}<0, \beta_{i}<0, \\
K_{2}(x, t)= & +\sqrt{\lambda_{2}} J_{0}\left[\sqrt{\lambda_{2}}(x-t)\right]+\lambda_{1} \int_{t}^{x} J_{0}\left[\sqrt{\lambda_{2}}(x-\xi)\right] d \xi+\frac{A_{i}(x-t)^{1-\alpha_{i}}}{\left(1-\alpha_{i}\right) \Gamma\left(1-\alpha_{i}\right)} \\
& -\frac{1}{\left(1-\alpha_{i}\right) \Gamma\left(1-\alpha_{i}\right)}(x-t)^{1-\beta_{i}} \int_{0}^{1} v^{1-\alpha_{i}} \frac{\partial}{\partial v}\left[A_{i} J_{0}\left[\sqrt{\lambda_{2}}(x-t)(1+v)\right]\right] d v \\
& +\frac{(x-t)^{1-\beta_{i}}}{\left(1-\beta_{i}\right) \Gamma\left(1-\beta_{i}\right)} \int_{0}^{1} v^{1-\beta_{i}} \frac{\partial}{\partial v}\left[J_{0}(x, t+(x-t) v)\right] d v, \quad 0<\alpha_{i}, \beta_{i}<1,
\end{aligned}
$$

here $A_{i}(x), f_{1}(x), f_{2}(x), K_{0}(x, t)$ depend on the given function, for the index implied summation from 1 to $n$. $\omega_{1}(0)$ is an unknown constant to be defined.

Thus, the solution of (48), taking account of (38), (36), (49), and (50), has the form

$$
\tau(x)=\int_{0}^{x} f_{1}(t)\left[1+\int_{t}^{x} R_{i}(s, t) d s\right] d t+\omega_{1}(0) \int_{0}^{x} f_{2}(t)\left[1+\int_{t}^{x} R_{i}(s, t) d s\right] d t,
$$

where $R_{i}(x, t)$ is the resolvent of the kernel $K_{i}(x, t)(i=1,2)$.

Hence, by virtue of the condition $\tau(1)=\varphi_{3}(0), w_{1}(0)$ are determined uniquely, since, using relations (47), (44), (45), and (51), $v(x)$ are determined uniquely.

After finding $\tau(x)$ and $v(x)$, the solution of problem $\mathrm{T}_{1}$ defined in $\Omega_{2}$ by a formula due to Cauchy [4] or Darboux (see (43)). For the construction of the solution BVP $A$ in $\Omega_{1}$ we pass to the auxiliary problem $A^{*}$ [13] for (39) and similarly to [13] we prove the uniqueness of the solution.

Problem $\mathrm{T}_{1}$ is uniquely solvable. Theorem 2 is proved. Analogously, one can prove the uniqueness of the solution problem $T_{1}$ in the case where $\lambda_{1}>0, \lambda_{2}<0$.

\section{Competing interests}

The author declares that they have no competing interests.

\section{Author's contributions}

The author read and approved the final manuscript.

\section{Acknowledgements}

The author is grateful to Professor B Islamov for drawing attention to these problems; she would also like to thank the anonymous reviewers for their valuable suggestions.

Received: 30 November 2013 Accepted: 28 August 2014 Published online: 25 September 2014

\section{References}

1. Gel'fand, IM: Some questions of analysis and differential equations. Usp. Mat. Nauk 14(3(87)), 3-19 (1959)

2. Nakhushev, AM: The Equations of Mathematical Biology. Vishaya Shkola, Moscow 301 p. (1995)

3. Nakhushev, AM: Loaded equations and their applications. Differ. Equ. 19(1), 86-94 (1983)

4. Tikhonov, AN, Samarskij, AA: Equations of Mathematical Physics. Nauka, Moscow (1977)

5. Sabytov, KB: Construction in an explicit form of solutions of the Darboux problems for the telegraph equation and their application in generalizing integral equations. Differ. Equ. 25(6), 1023-1032 (1990)

6. Bateman, G, Erdelji, A: Higher Transcendent Functions. Nauka, Moscow (1966) 
7. Djuraev, TD: Boundary Value Problems for the Problems for the Mixed Type Equations. Fan, Tashkent (1971)

8. Salakhitdinov, MS: Equations of Mixed-Composite Types. Fan, Tashkent 155 p. (1974)

9. Baltaeva, UI, Islomov, BI: Boundary value problems for the loaded equations of the hyperbolic and mixed types. Ufim. Mat. Zh. 3(3), 15-25 (2011)

10. Krasnov, ML: Integral Equations. Introduction to the Theory. Nauka, Moscow (1975)

11. Baltaeva, UI, Islomov, BI: Boundary value problems for the classical and mixed integro-differential equations with Riemann-Liouville operators. Int. J. Partial Differ. Equ. 2013, Article ID 157947 (2013)

12. Dzhurayev, TD, Sopuev, A, Mamayhonov, M: Boundary Value Problems for the Problems for the Parabolic-Hyperbolic Type Equations. Fan, Tashkent (1986)

13. Eshmatov, BE, Karimov, ET: Boundary value problems with continuous and gluing conditions for parabolic-hyperbolic type equations. Cent. Eur. J. Math. 5(4), 741-750 (2007)

doi:10.1186/s13661-014-0211-6

Cite this article as: Baltaeva: Solvability of the analogs of the problem Tricomi for the mixed type loaded equations with parabolic-hyperbolic operators. Boundary Value Problems 2014 2014:211.

\section{Submit your manuscript to a SpringerOpen ${ }^{\circ}$ journal and benefit from:}

- Convenient online submission

- Rigorous peer review

- Immediate publication on acceptance

- Open access: articles freely available online

- High visibility within the field

- Retaining the copyright to your article 